\title{
3-D Segmentation and Reconstruction of the Retinal Vasculature from Spectral-Domain Optical Coherence Tomography
}

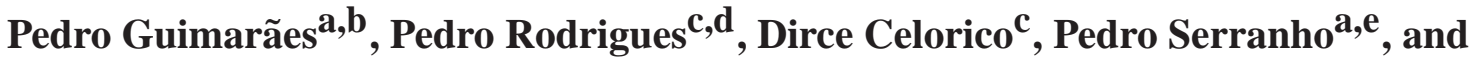 \\ Rui Bernardes ${ }^{a}$ \\ a IBILI - Institute for Biomedical Imaging and Life Sciences, Faculty of Medicine, University of Coimbra, \\ Coimbra, Portugal. \\ $\mathrm{b}$ Department of Information Engineering, University of Padova, Padova, Italy \\ c AIBILI - Association for Innovation and Biomedical Research in Light and Image, Coimbra, Portugal \\ d ISR — Institute of Systems and Robotics, University of Coimbra, Coimbra, Portugal. \\ e Mathematics Section, Department of Science and Technology, Universidade Aberta, Lisbon, Portugal.
}

\begin{abstract}
In this work we reconstruct the 3-D shape and location of the retinal vascular network from commercial spectral-domain (SD) optical coherence tomography (OCT) data. The 2-D location of retinal vascular network on the eye fundus is obtained through SVM classification of properly defined fundus images from OCT data, ${ }^{1}$ taking advantage of the fact that on standard SD-OCT the incident light beam is absorbed by haemoglobin, therefore creating a shadow cast on OCT signal below each perfused vessel. The depth-wise location of the vessel is obtained as the beginning of the shadow. The classification of crossovers and bifurcations within the vascular network is also addressed. We illustrate the feasibility of the method in terms of vessel calibre estimation and the accuracy of bifurcations and crossovers classification.
\end{abstract}

Keywords: Optical coherence tomography, Vascular system, Retina.

\section{Introduction}

The retina is regarded as a window to the cardiovascular system. Changes in the retinal microvasculature have been found to be related with several cardiovascular ${ }^{2-4}$ and cerebrovascular ${ }^{4-9}$ out- $^{-}$ comes, among other. ${ }^{10-12}$ These evidences turn the automatic detection of retinal blood vessels a key step in this area of research. The quantitative description of the detected retinal vasculature, can be and has been used to establish the association between retinal vascular properties and clinical and subclinical outcomes, thus providing tools to the clinician for an objective diagnosis.

Extensive work has been done in this field, mainly based on two widely used ocular imaging modalities: color fundus photography (CFP) and fluorescein angiography (FA). Vascular properties such as tortuosity or bifurcation angles computed from these 2-D fundus images have been associated with several diseases. However, the computation of such properties is incomplete due to the projection to a 2-D plane. A robust method to segment the human retinal vascular network in 3-D would be a valuable tool and a significant leap forward to fully understand the pathophysiology of several diseases.

Optical coherence tomography (OCT) is an imaging modality capable of noninvasively image the microstructure of tissue in vivo and in situ. ${ }^{13,14}$ Overtime, it became an important tool in the diagnosis of ocular pathologic conditions. It has been used extensively in clinical research and is becoming common in the clinical practice. The principle, based on the backscattering of lowcoherence light, is now extensively described in the literature. ${ }^{13,14}$

On standard spectral-domain OCT (SD-OCT) scans, the retinal blood vessels are not directly visible. Instead two signatures emerge in the OCT signal. One relates to the fact that haemoglobin absorbs the infrared light. Consequently, backscattering at the structures below perfused vessels is 


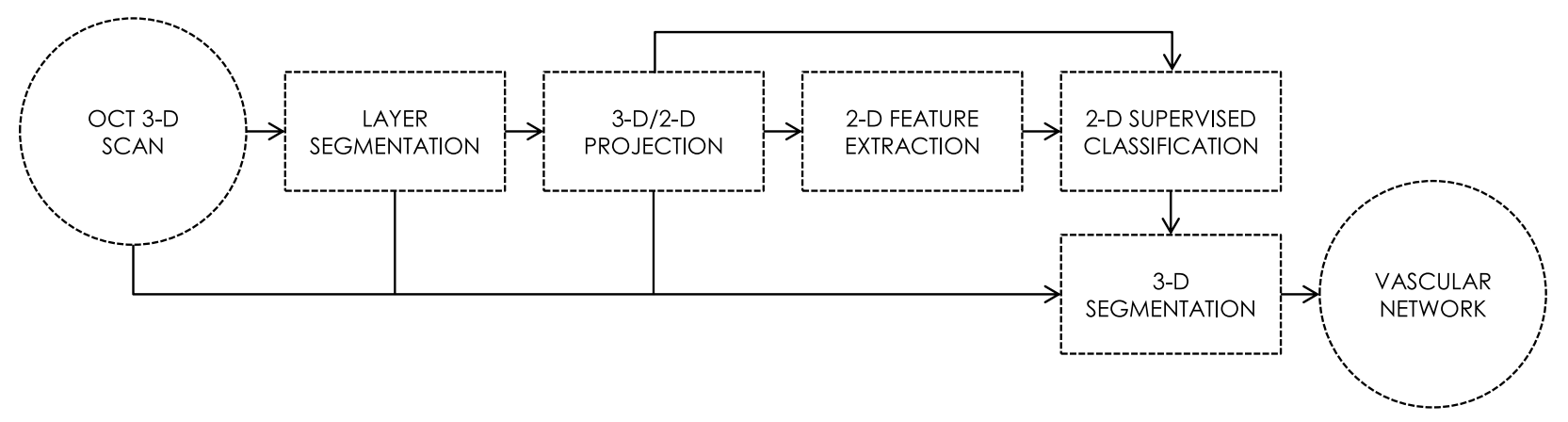

Fig 1 Flowchart representing the global workflow of the algorithm.

highly attenuated. ${ }^{13,15,16}$ This effect is well known and has been used to obtain the 2-D vascular network from the 3-D OCT data. ${ }^{17-19}$ While this is a major advantage for 2-D segmentation due to the significant contrast on the retinal pigment epithelium (RPE), the 3-D segmentation of the retinal vasculature requires additional information. The other signature is a diffuse hyper-reflectivity on the vessel itself.

Approaches for 3-D retinal vasculature segmentation in the literature are limited in number. ${ }^{20-22}$

In this work we describe a fully-automatic method for the 3-D segmentation of the vascular network of the human retina from standard Cirrus HD-OCT (Carl Zeiss Meditec, Dublin, CA, USA) data and the framework towards its reconstruction.

\section{Workflow and Background Work}

Following a preliminary study by our research group, ${ }^{1}$ the 3 -D OCT scan is projected to a 2-D ocular fundus reference image. Each pixel on this image translates into an A-scan on the OCT volume. This reference is then used to compute features that are able to discriminate each pixel into the vessel or non-vessel groups. ${ }^{23}$ The A-scans whose pixels were classified into the vessel group, i.e., A-scans that intersect blood vessels, are then processed to determine the depth-wise location of the vessel. Fig. 1 shows the global workflow of the algorithm and Fig. 2 shows a graphical depiction of the process.

Throughout this paper, the following coordinate system for the OCT data will be used: $x$ is the nasal-temporal direction, $y$ is the superior-inferior direction, and $z$ is the anterior-posterior direction (depth).

\subsection{Retinal Layer Segmentation}

The very first step consists of determining the depth coordinates of three interfaces: $Z_{1}$ the inner limiting membrane (ILM), $Z_{2}$ the junction between the inner and outer photoreceptor segments (IS/OS), and $Z_{3}$ the interface between the retinal pigment epithelium (RPE) and the choroid. These interfaces are typically easiest ones to identify.

The retinal layer segmentation process itself will not be discussed here in great detail. In the one hand, it does not greatly affect the quality of the results of the final 3-D vascular segmentation and, on the other hand, there is a strong background of published work in this area. ${ }^{24,25}$ Each B-scan is first filtered with a 2-D Gaussian derivative filter. The rational is that $Z_{1}, Z_{2}$, and $Z_{3}$ interfaces correspond the strongest intensity transitions in a normal retina. A threshold is then applied. Finally 3-D gradient smoothing is applied to the obtained surfaces. 


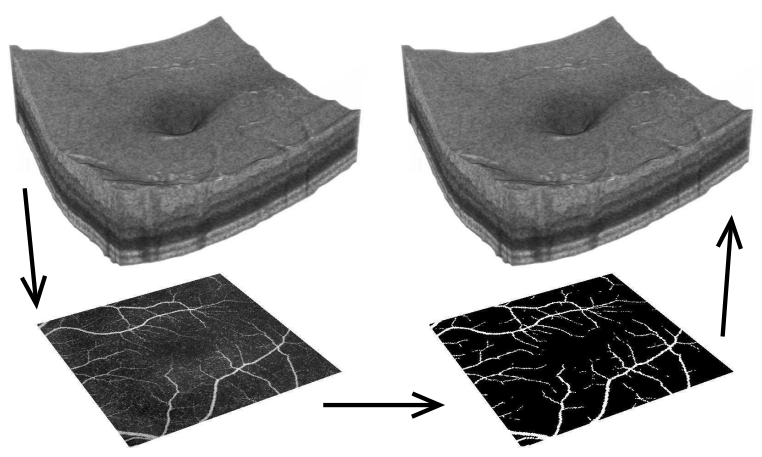

Fig 2 Projection of a 3-D macular OCT scan to a 2-D fundus reference image by mapping the shadows casted due to light absorption. The binary image (from the classification of the pixels in the 2-D reference) is then used to aid in the identification of the depth location of the vessels.

\subsection{Ocular Fundus Reference Images}

As noted, light absorption by haemoglobin is responsible for the decrease in light backscattering beneath perfused vessels. The segmentation process herein takes advantage of this effect by computing a set of 2-D fundus reference images from the 3-D OCT data (Fig. 2). A study was conducted to identify the fundus reference images that provide the best discrimination between the vascular network and the background. ${ }^{26}$ Four potential reference images were evaluated: ${ }^{26}$ the mean value fundus (MVF), the expected value fundus (EVF), the error to local median (ELM), and the principal component fundus (PCF).

The PCF image is computed through principal component analysis (PCA) as the principal component of the MVF, EVF, and ELM images. The interfaces at coordinates $Z_{2}$ and $Z_{3}$ are needed for this stage of the process. It was demonstrated that the PCF image provides the greatest extension of the vascular network (equivalent to that achieved with CFP) and the best contrast amongst the other fundus reference images. ${ }^{26}$

\subsection{Feature Computation and 2-D Classification}

To obtain the 2-D vascular network segmentation we resort to an approach previously publish by Rodrigues et al. ${ }^{23}$ For clarity, we shortly describe in this section the used algorithm and the obtained results.

All four 2-D fundus reference images computed from OCT volumes (MVF, EVF, ELM, and $\mathrm{PCF}$ ) were used as features in the classification process. Additional features were computed from the PCF image. Specifically, intensity-based features, local-phase features, and features computed with Gaussian-derivative filters, log-Gabor and average filter banks, and band-pass filters. ${ }^{23} \mathrm{~A}$ supervised learning algorithm (support vector machine) was then used to train and classify each A-scan of the OCT volume $V$ into vessel or non-vessel A-scans, i.e., to discriminate between A-scans that cross retinal blood vessels and the remaining A-scans. Formally, we define the set $\mathcal{A}=\left\{A_{i}():. \forall i \in \mathcal{U}\right\}$ of A-scans $A_{i}()=.V\left(x_{i}, y_{i},.\right)$ that cross vessels. The set $\mathcal{U}$ is defined as the indexes of A-scans that cross vessels.

The process proved to cope with OCTs of both healthy and diseased retinas. It achieved good results for both the macular and optic nerve head regions. For a set of macular OCT scans of healthy subjects and diabetic patients, the algorithm achieved $98 \%$ accuracy, $99 \%$ specificity, and 


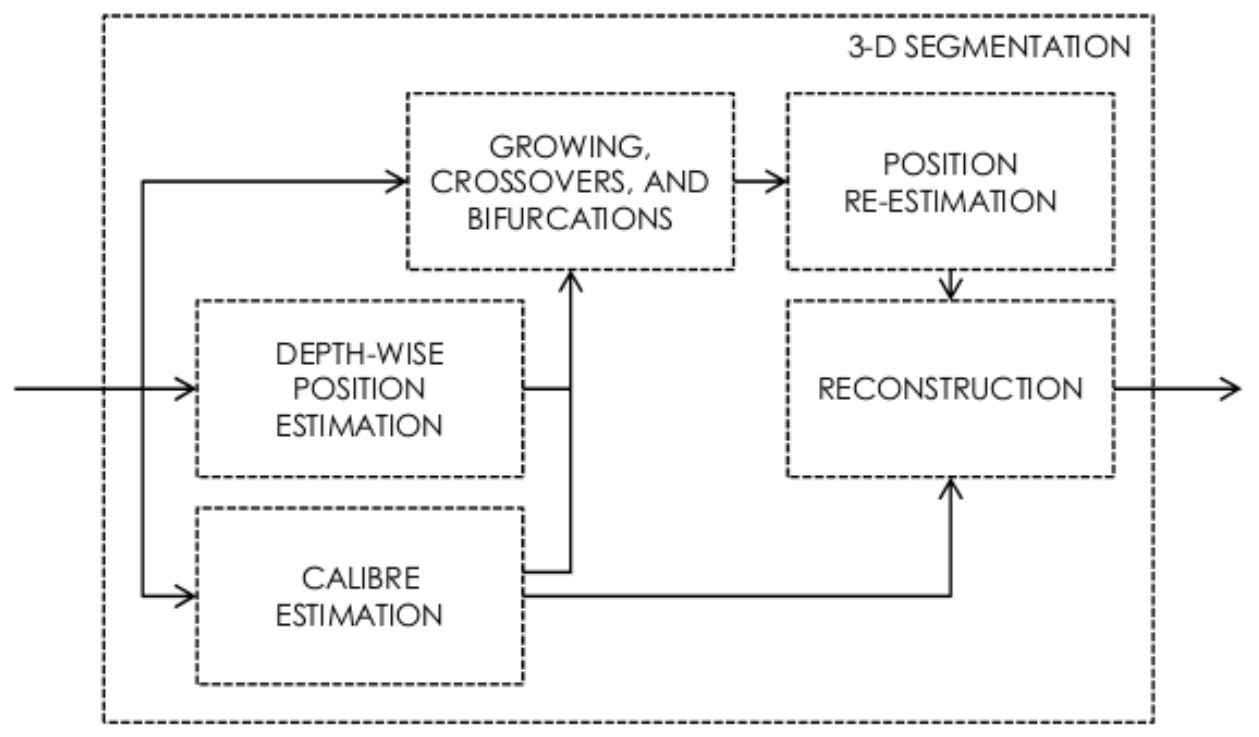

Fig 3 Flowchart for the 3-D segmentation block of Fig. 1. The main block inputs are the OCT volume, the segmented layers, the 2-D ocular fundus reference image, and the binary image (Fig. 2).

$83 \%$ sensitivity. For both groups the algorithm compared favourably to the inter-grader agreement. $^{23}$

\section{3-D Segmentation and Reconstruction}

The core of the work presented in this paper addresses the issues of estimating vessels diameters and their depth-wise location within the OCT volume, and the 3-D modelling of the vascular network from the human retina, dealing with crossovers and bifurcations. The workflow of this process can be found in Fig. 3.

\subsection{Vessel Calibre Estimation}

The lateral resolution of OCT combined with the optical properties of the vessel walls do not allow for their direct observation. As such, vessel calibre can only be estimated from the shadow casted over the RPE due to light absorption by haemoglobin. Due to the aforementioned reasons (section 2.2), the PCF ocular fundus reference image is the natural choice for the vessel calibre estimation. It is thus clear that the estimated calibre is below the real calibre and that this effect is less significant for larger vessels, closer to the optic disc, and become more important close to the fovea as vessels become thinner. In addition, the estimated calibre can only be achieved in the $x y$ plane and we herewith assume them to have a circular cross-section.

There is a wealth of literature on methods to compute retinal vessel width from 2-D ocular fundus images. These were tailored for imaging modalities such as CFP and FA. ${ }^{27-29}$ These methods rely on the estimation of the cross-section with respect to vessel centrelines and have to deal with the rough definition of vessel borders due to the regular sampling of the digital imaging modalities. $^{30,31}$ This effect is much more prominent on OCT rendering these methods inappropriate for this modality. 

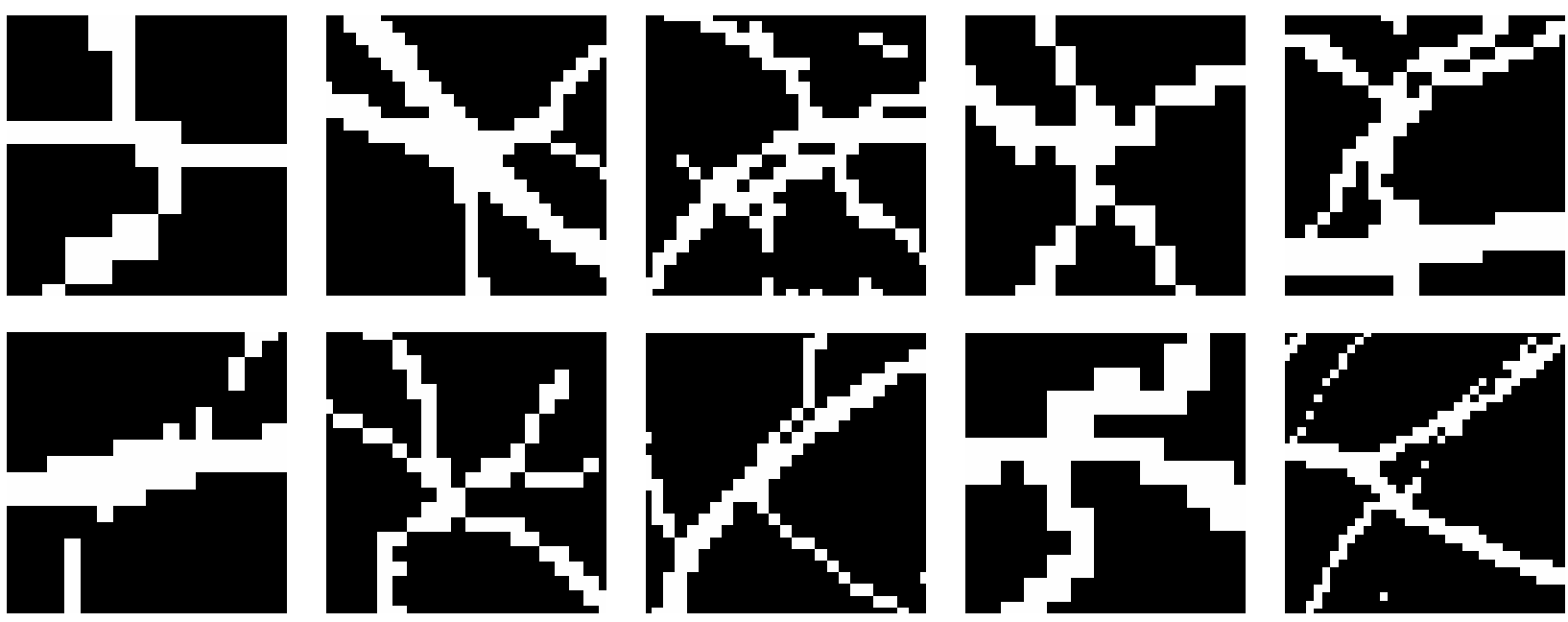

Fig 4 Examples of 2-D vessel binary maps cropped to bewildering regions of crossovers and/or bifurcations.

Log-Gabor wavelets are routinely used for vessel enhancement and detection. ${ }^{32}$ These are created by combining a radial and an angular component (in the frequency space), determining the scale and the orientation of the filter, respectively. In the time domain, the filter is composed by an even (real part) and an odd (imaginary part) kernels.

To determine the retinal vessels calibre, the PCF image is filtered with a bank of log-Gabor even kernels, each with a unique orientation-scale pair. This bank covers a wide range of scales and orientations to fully describe the whole vascular network. At each vessel pixel $\operatorname{PCF}\left(x_{i}, y_{i}\right)$, with $i \in \mathcal{U}$, the log-Gabor even kernel whose orientation-scale pair better matches the vessel orientation and calibre, generates the highest response. The scale of the kernel with maximal response can then be translated into the calibre of the vessel on that pixel.

\subsection{Bewildering Regions}

The bewildering regions are subsets of neighbouring vascular network A-scans where the path of the vessels is unclear from the 2-D segmentation due to bifurcation and/or crossovers (Fig.4). To determine these regions several binary morphological operations are used. These regions require further processing (see section 3.4).

First, endpoints in the vicinity of one another on the binary vascular fundus image are bridged thus redefining the binary image (the set of vessel points $\mathcal{U}$ ). This updated image is then skeletonized and isolated points are removed. Finally, the bewildering regions are defined as the dilation of branch points and these are removed (erased) from the skeleton image. In consequence, all potential bifurcations and crossovers are left to be re-linked adequately. This new set of points defines the set $\mathcal{U}^{\text {skel }}$ (Fig. 5).

The set $\mathcal{E}_{c} \subset \mathcal{U}^{\text {skel }}$ of endpoints on each bewildering region $c$, with $\mathcal{E}_{c} \in\left\{\mathcal{E}_{1}, \mathcal{E}_{2}, \ldots\right\}$, will then be used to look for the most plausible linking configuration of that region (section 3.4).

\subsection{Vascular Network Depth-Wise Position}

As stated, retinal blood vessels are not directly visible in standard OCT data. Typically, vessels on OCT appear as hyper-reflective regions followed posteriorly by the shadowing of structures beneath due to light absorption by haemoglobin in perfused vessels. 


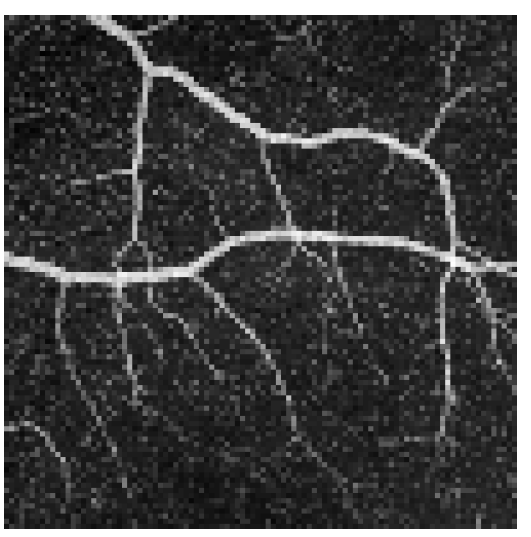

(a)

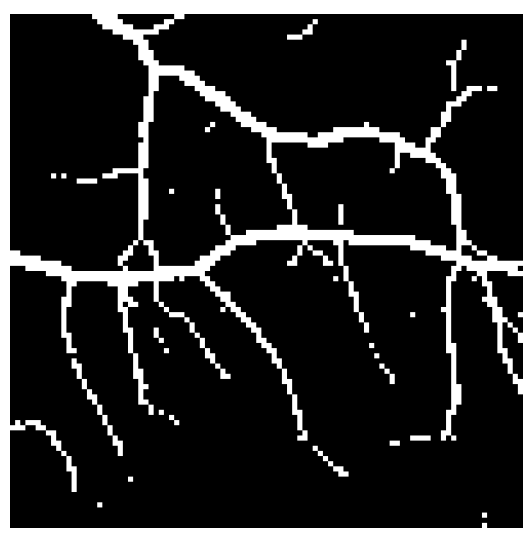

(b)

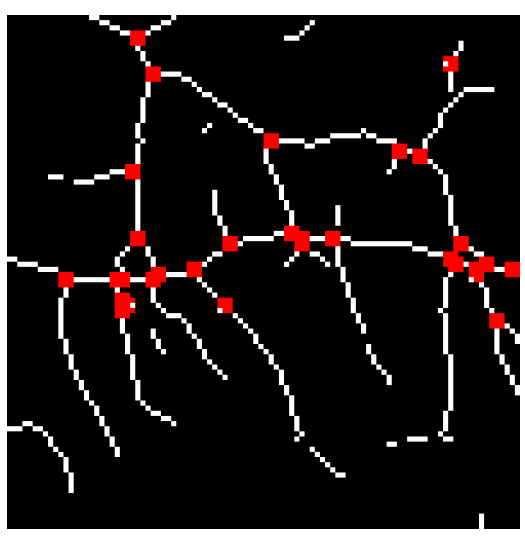

(c)

Fig 5 Detail of a principal component fundus reference image (a) and the respective binary maps of the 2-D classification $\mathcal{U}(\mathrm{b})$ and of the vessel centrelines (with the removed bewildering regions in red) $\mathcal{U}^{\text {skel }}$ (c).

In the previous section, all A-scans were classified as vessel or non-vessel A-scans. Only Ascans from the centreline of the vessel are processed to search for the depth-wise location of the vessel, using the following methods.

\subsubsection{Principal Component Analysis}

PCA is herewidth used to enhance similar information between neighbour vessel A-scans in the principal component.

For each vessel centreline A-scan $\left(\mathcal{A}^{\text {skel }}=\left\{A_{i}():. \forall i \in \mathcal{U}^{\text {skel }}\right\}\right)$, a circular region in the $x y$ plane centred at $\left(x_{i}, y_{i}\right)$ with radius $r$ is defined. The set of A-scans in each vessel and non-vessel region are used to compute two new profiles, $A^{\text {vessel }}$ and $A^{\text {non-vessel }}$, respectively, as follows.

Every A-scan within the defined region is interpolated to account for differences in retinal thickness, from the IML $\left(Z_{1}\right)$ to the IS/OS $\left(Z_{2}\right)$. One should bear in mind that the whole volume was previously flattened by the IS/OS layer ${ }^{26}$ and, as such, $Z_{2}$ is now equal for all A-scans.

Each profile in $\mathcal{A}^{\text {vessel }}=\left\{A_{i}^{\text {vessel }}():. \forall i \in \mathcal{U}^{\text {skel }}\right\}$ is computed as the principal component of the selected A-scans $A_{j}$ such that

$$
A_{j}:\left\|\left[x_{i}-x_{j}, y_{i}-y_{j}\right]\right\|<r, \forall\{i, j\} \in \mathcal{V}_{k}
$$

where $\mathcal{V}_{k} \subset \mathcal{U}$ is the set of indexes of the A-scans that constitute the vessel $k$ and $\|$.$\| is the$ usual Euclidean norm. On the other hand, each profile in $\mathcal{A}^{\text {non-vessel }}=\left\{A_{i}^{\text {non-vessel }}():. \forall i \in \mathcal{U}^{\text {skel }}\right\}$ is computed as the principal component of the selected A-scans $A_{j}$ such that

$$
A_{j}:\left\|\left[x_{i}-x_{j}, y_{i}-y_{j}\right]\right\|<r, \forall j \notin \mathcal{U} \text {. }
$$

At the first iteration the sets $\mathcal{V}_{k} \in\left\{\mathcal{V}_{1}, \mathcal{V}_{2}, \ldots\right\}$ are vessel segments delimited by bewildering regions. However, after defining the actual links between segments, the depth-wise position can be recomputed to improve the estimation at the bewildering regions (Fig. 3).

Although the radius $r$ was selected empirically following visual inspection of the final 3-D segmentation, it is now kept fixed for the processing of all the examples. 


\subsubsection{Local Difference Profiles}

To estimate the $z$ (depth) coordinate of the vessel at each point of the centreline, the difference between the vessel $\left(A_{i}^{\text {vessel }}\right)$ and the non-vessel $\left(A_{i}^{\text {non-vessel }}\right)$ profiles are computed for each $i \in \mathcal{U}^{\text {skel }}$. This operation results in a difference profile with two clear signatures, one due to the presence of the hyper-reflectivity and the other due to the shadow in vessel A-scans only.

Although the vessel walls are not seen in the difference profile, one can estimate their position (and, therefore, the vessel centre) by taking advantage of its diameter estimated in section 3.1. A moving average filter $h(x, d)$ with size $d$ allows for the estimation of the center of the vessel by

$$
c_{i}=\underset{z}{\operatorname{argmax}}\left[\left(A_{i}^{\text {vessel }}(z)-A_{i}^{\text {non-vessel }}(z)\right) * h\left(z, s_{i}\right)\right]
$$

where $s_{i}$ is the estimated calibre at the $i$-th A-scan, $*$ is the convolution operator, and $c_{i}$ is restricted to be in the interval $\left[Z_{1}+s_{i} / 2, Z_{2}-s_{i} / 2\right]$.
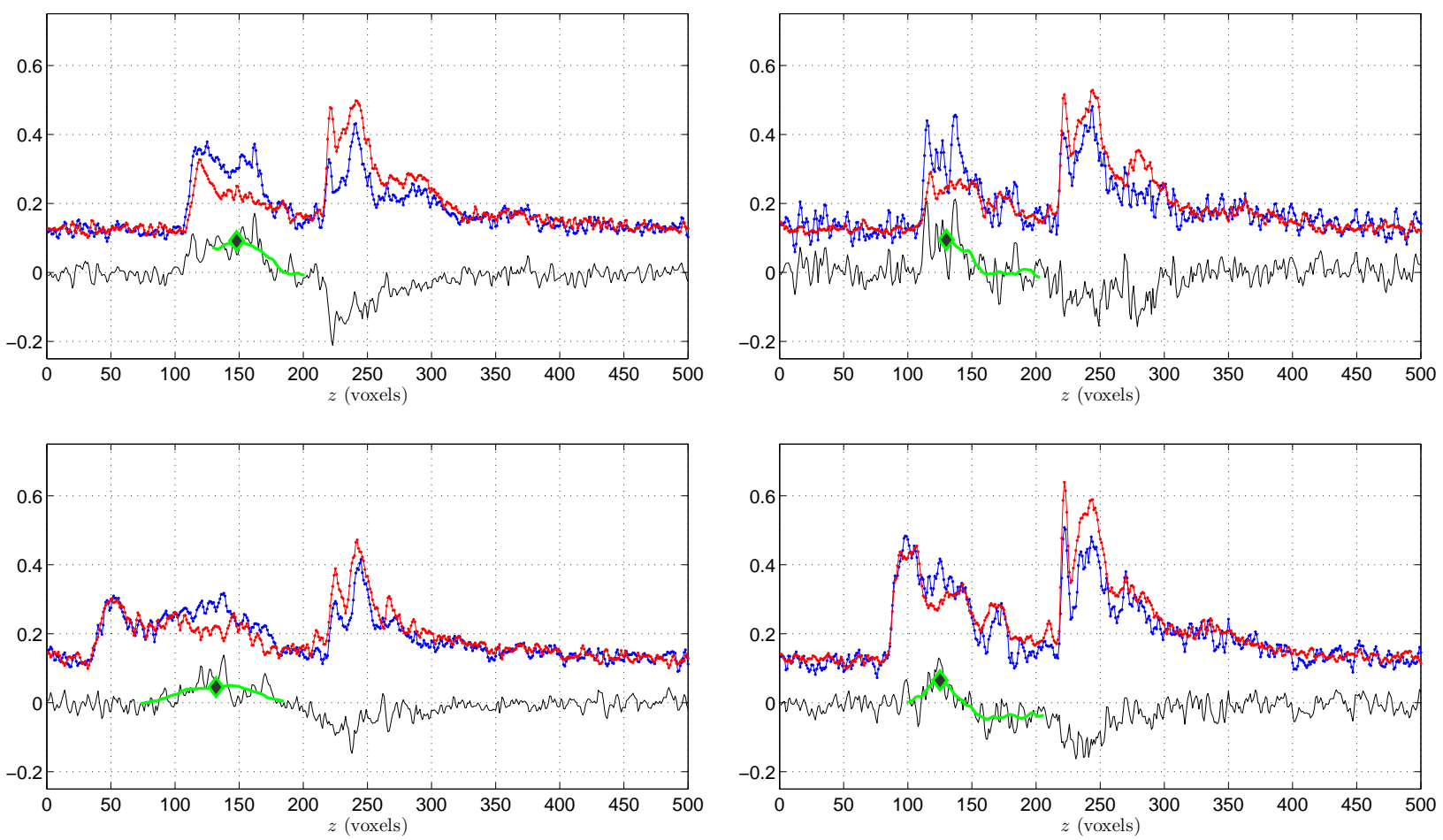

Fig 6 Computation examples of the depth coordinate of different blood vessel at a centreline point $i$. The two profiles on the top of each plot are the vessel profile $A_{i}^{\text {vessel }}$ (blue) and the non-vessel profile $A_{i}^{\text {non-vessel }}$ (red). The difference between the two (black profile) is shown on the bottom. The difference profile is filtered at the region of interest (green) and the depth coordinate of the vessel on that point is taken as the location of the maximum of the filtered difference.

\subsection{Bifurcations and Crossovers}

Vessel tracking in OCT is quite different from other imaging modalities. Due to the low sampling of OCT systems, compared to imaging modalities as CFP and FA, most vessels are just one to two pixels wide and the bewildering regions present many alternatives for the linking process in 
windows just a few pixels wide (Fig. 4). Furthermore, since we use the shadow to locate the blood vessels, the intensity of different vessels on the fundus reference images do not differ sufficiently to tell them apart.

As a pre-processing step before linkage, we screen all the possible links to find those that are unlikely and to find some links that were not established in the 2-D classification that could help solving the bewildering region (the so-called growing links). All possible links are then subject to thresholding by length, angle between linked vessel segments, and angle between each vessel segment and the link itself.

For each bewildering region $c$, a cost $\phi_{i j}$ is assigned to every link $l_{i j}$ between every two points, $\left(x_{i}, y_{i}, z_{i}\right)$ and $\left(x_{j}, y_{j}, z_{j}\right)$, such that $\{i, j\} \in \mathcal{E}_{c}$ and $i<j$, as

$$
\phi_{i j}=\left(\alpha_{i j}+\beta_{i j}+\gamma_{i j}\right)\left(\alpha_{j i}+\beta_{j i}+\gamma_{j i}\right)
$$

with

$$
\begin{aligned}
\alpha_{i j} & =\frac{2}{\pi}\left|\theta_{i}^{x y}-\arctan \left(\frac{y_{j}-y_{i}}{x_{j}-x_{i}}\right)\right| \\
\beta_{i j} & =1-\frac{\min \left(s_{i}, s_{j}\right)}{\max \left(s_{i}, s_{j}\right)} \\
\gamma_{i j} & =\frac{2}{\pi}\left|\theta_{i}^{z}-\arctan \left(\frac{z_{j}-z_{i}}{\left\|\left[x_{i}-x_{j}, y_{i}-y_{j}\right]\right\|}\right)\right|
\end{aligned}
$$

with arctan and the difference of angles mapped to the interval $[-\pi / 2, \pi / 2]$, and $\theta^{x y}$ and $\theta^{z}$ are, respectively, the orientation of the vessel in the $x y$ plane and in $z$.

At this point we shall define all configurations $\mathcal{C}_{k} \in\left\{\mathcal{C}_{1}, \mathcal{C}_{2}, \ldots\right\}$ as sets of links $l_{i j}$ subject to the following constraints:

- the set of links contains the link with least cost $\left(l_{i j}:(i, j)=\operatorname{argmin}_{i, j} \phi_{i j}\right)$;

- all end-points have to be linked, except points that need vessel segmentation growing to link between them;

- it has only one link by vessel segmentation growing;

- the configuration does not result in intersections or loops in the same vessel.

Generally, the set $\left\{\mathcal{C}_{1}, \mathcal{C}_{2}, \ldots\right\}$ will enclose all possibilities for crossovers and bifurcations.

The cost of the configuration $\mathcal{C}_{k}$ is then computed as

$$
\Phi_{k}=\sum_{(i, j): \forall l_{i j} \in \mathcal{C}_{k}} \phi_{i j}
$$

From the group of many feasible combinations, i.e. ignoring the ones rendering loops within the same vessel, the solution presenting the lowest overall cost is chosen. 


\subsection{3-D Reconstruction}

Vessel centrelines in the $x y$ plane (section 3.2) are combined with the estimated vessel centrelines of section 3.4 to render the 3-D skeleton on the vascular network.

In this work we assume vessels to have a local tubular structure whose centrelines are defined by the 3-D skeleton and the diameter is estimated from the fundus reference image ( $x y$ plane). In this way, cylinders and cone-like structures are the fundamental components from which the 3-D vascular network is built.

\subsubsection{Vessels Path Interpolation}

The combination of vessel diameter and low OCT sampling results in vessels is poorly defined in the fundus reference. In addition, at vessel crossovers the basic assumptions for the determination of the vessel depth location is no longer verified, except for the top one. That is, the vessels below the top one do not present the clear signatures (vessel hyper-reflectivity and shadow) since they lay on the shadow casted by the vessel above. In this way, interpolation is mandatory and is performed under the assumption that vessel do not present discontinuities or sharp edges, i.e. they are continuous with respect to the first derivative, in any of the 3 dimensions. Under these assumptions, the vascular network is built based on OCT data (control points) making use of cubic spline interpolation.

\subsubsection{Delaunay Triangulation}

Vessel reconstruction is achieved by Delaunay triangulation. At each vessel centreline location, the tangent to the path, defined by the centreline points, is computed and the respective normal plane determined. The cross-section of the vessel is approximated by a set of points in this crosssectional plane within the circumference centred at the vessel centreline and a diameter equal to the estimated vessel diameter (Fig. 7). Adjacent circumferences are later connected using the Delaunay triangulation in 3-D. The quality of the final reconstruction is directly dependent on the number of triangles, which in turn depends on the circumference sampling and the gap between estimated vessel cross-sections.

\section{Results}

OCT macular scans of 15 eyes from healthy subjects and eyes from patients diagnosed with type 2 diabetes mellitus (Early Treatment Diabetic Retinopathy Study levels levels 10 to 35) were used as test bed for the proposed methodology. All OCT scans were gathered from our institutional database and were collected by a Cirrus HD-OCT (Carl Zeiss Meditec, Dublin, CA, USA) system. These eyes were also imaged by CFP (Zeiss FF 450 system) and/or FA (Topcon TRC 50DX, Topcon Medical Systems, Inc., Oakland, NJ, USA).

The results obtained for the 3-D vascular segmentation are now presented in three parts: the vessel calibre estimation, the bewildering regions decision and the reconstruction and $z$ position of the vessels. 


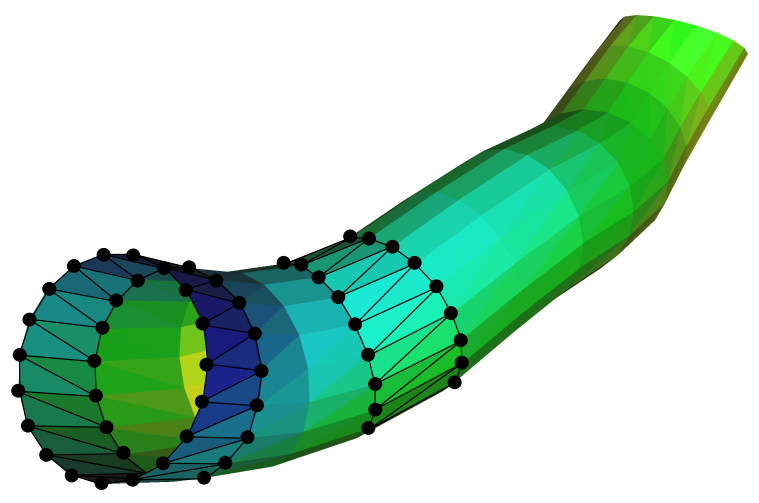

Fig 7 Illustration of a local reconstruction of a vessel, based on Delaunay triangulation. Several points over the centreline are chosen and equally distant points (with distance equal to the estimated radius of the vessel in the $x y$ plane) inn the respective orthogonal plane to the centreline are considered for the 3-D triangulationprocess of reconstruction.

\subsection{Vessel calibre}

The automated vessel calibre estimation was compared with the assisted estimates made by three graders $\left(G_{1}, G_{2}\right.$, and $\left.G_{3}\right)$.

Five vessel segments per OCT eye scan were randomly chosen, each with a minimum of 21 pixels long along the centreline. Graders were instructed to use a software tool. Since the lateral sampling of OCT is relatively low, the graders would not be able to simply mark the vessel boundaries, as it is common practice for CFP [33]. Instead, the tool provided, for any given calibre, a sub-pixel continuous marking of the boundaries (for that particular calibre) of the whole vessel segment against the PCF image. Several diameters with increments of 0.2 pixels were tested by the graders to choose the diameter that best fits the data. The result is a set of several calibre measurements (in pixels) for each randomly chosen vessel segment.

For the comparison, two metrics were used, the absolute and the relative differences as defined, respectively, by

$$
\begin{aligned}
& D^{a}(G, \hat{G})=|G-\hat{G}| \\
& D^{r}(G, \hat{G})=|G-\hat{G}| / \hat{G}
\end{aligned}
$$

where $G$ is the data to test and $\hat{G}$ is the ground truth, here considered as the average of all graders (Table 1).

Table 1 shows the average metric results by comparing the automatic estimation $(S)$ to the average grading of all graders and each grader to average grading of the other two, as a measure of inter-grader agreement. Fig. 8 shows the relative difference metric for several ranges of diameters for the same comparisons. As expected, smaller vessels render a higher variability as shown by the 
Table 1 Comparison between the automatic calibre estimation $(S)$ and each manual grading $\left(G_{1}, G_{2}\right.$, and $\left.G_{3}\right)$, by the mean and standard deviation (SD) values for the absolute difference $D^{a}$ (pixels) and the relative difference $D^{r}$ between the result $G$ and $\hat{G}$.

\begin{tabular}{lllll}
\hline Metric & $G$ & $\hat{G}$ & Mean & SD \\
\hline$D^{a}(G, \hat{G})$ & $S$ & $1 / 3\left(G_{1}+G_{2}+G_{3}\right)$ & 0.311 & 0.208 \\
& $G_{1}$ & $1 / 2\left(G_{2}+G_{3}\right)$ & 0.494 & 0.240 \\
& $G_{2}$ & $1 / 2\left(G_{1}+G_{3}\right)$ & 0.187 & 0.153 \\
& $G_{3}$ & $1 / 2\left(G_{1}+G_{2}\right)$ & 0.406 & 0.231 \\
\hline$D^{r}(G, \hat{G})$ & $S$ & $1 / 3\left(G_{1}+G_{2}+G_{3}\right)$ & 0.213 & 0.173 \\
& $G_{1}$ & $1 / 2\left(G_{2}+G_{3}\right)$ & 0.299 & 0.168 \\
& $G_{2}$ & $1 / 2\left(G_{1}+G_{3}\right)$ & 0.122 & 0.110 \\
& $G_{3}$ & $1 / 2\left(G_{1}+G_{2}\right)$ & 0.337 & 0.266 \\
\hline
\end{tabular}
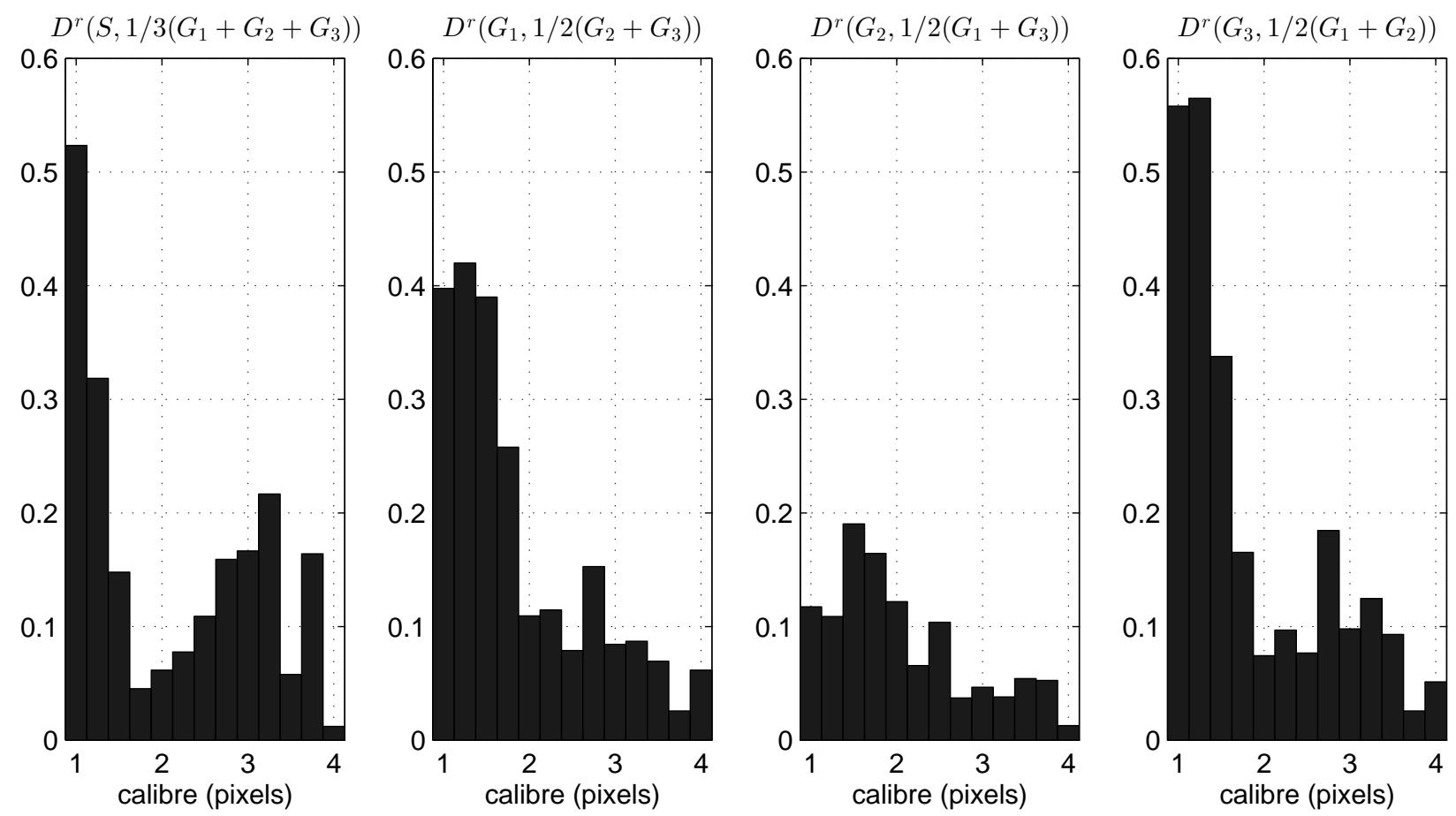

Fig 8 Average relative differences for the automatic estimation $(S)$ and manual gradings $\left(G_{1}, G_{2}\right.$, and $\left.G_{3}\right)$ displayed by calibre range.

histograms. Fig. 9 shows both the manual and automatic determination of vessel boudaries over the PCF fundus reference image.

\subsection{Bewildering Regions}

At bifurcation and crossover locations, the continuity of each vessel is hard to detect even for a human grader, naturally depending on the number of possibilities for each region. This demonstrated to be a very demanding task for the automatic process. For very difficult cases or whenever graders decided, they could access either a fluorescein angiography or color fundus photography, or both, according to the respective availability in our database. In general, the access to these 

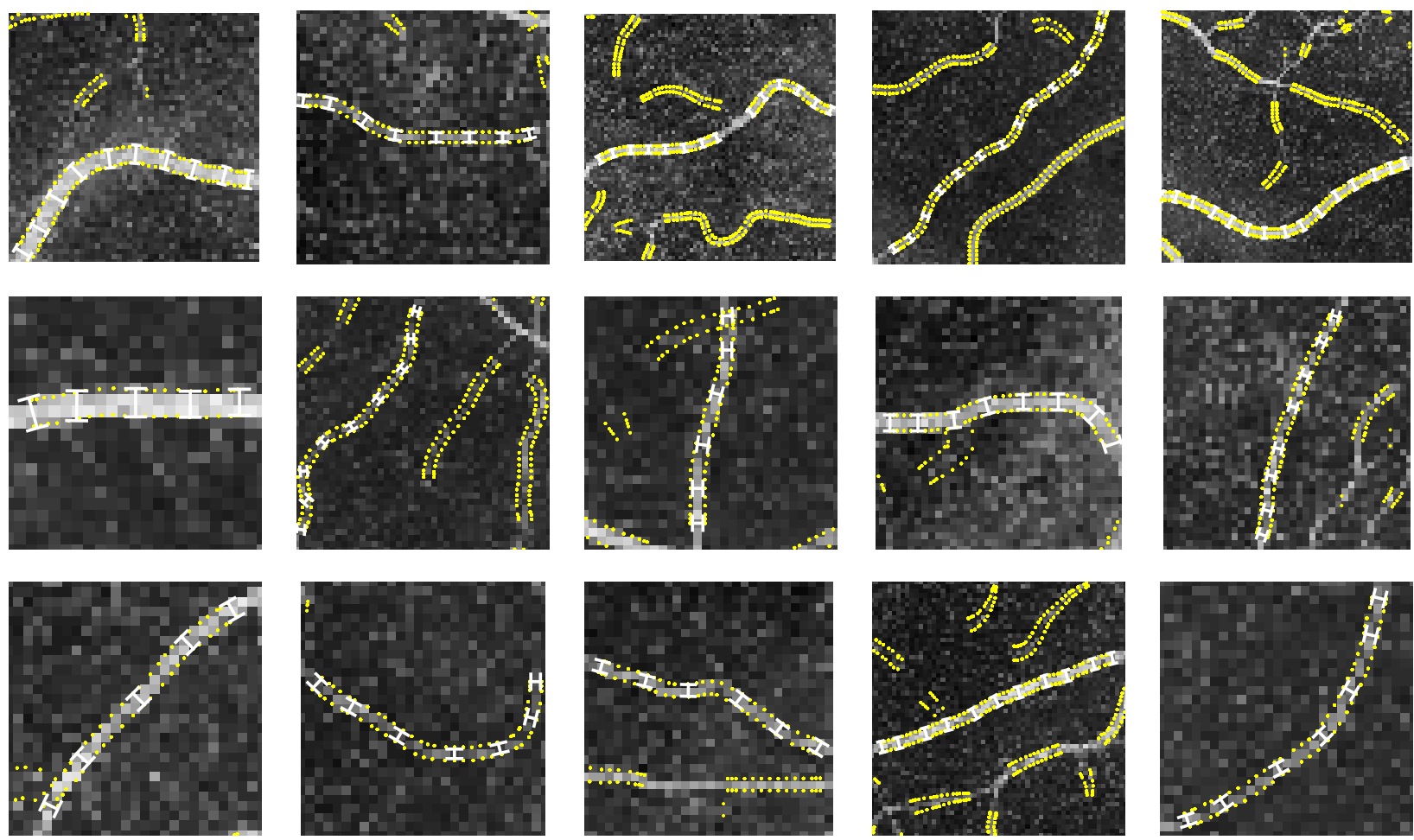

Fig 9 Representative results for the diameter estimation. The manual gradings are marked with (white) $\vdash \dashv$ and automatic estimation is marked with the (yellow) dotted line.

complementary imaging modalities proved to be frequently required for this task. Three metrics were used to assess the accuracy of the automated system in establishing the correct links between vessel segments at bewildering regions. These are: the point accuracy (indicates the percentage of points in $\mathcal{E}_{c}$ currectly grouped), the linking accuracy (the percentage of links correctly connected and nonlinks correctly unconnect, i.e., the percentage of true positives and false negatives) and, the percentage of bewildered regions correctly classified.

The results are shown in Figs. 10 and 12.

\subsection{Vascular Network Depth-Wise Position}

The low visibility of vessel markers (such as the shadow) renders the manual detection of blood vessels in OCT B-scans a hard task. Two graders $\left(G_{4}\right.$ and $\left.G_{5.1}\right)$ were instructed to mark, at 50 randomly selected vessel A-scans, the position where the shadow of the vessels begin. Some restrictions were imposed to the random selection to guarantee an unbiased evaluation: the same vessel could not be selected twice and no more than five A-scans per exam were possible. Furthermore, very small vessels (less than two pixels in radius) were excluded as the graders found very difficult to evaluate them. Both graders evaluated the same A-scans so that inter-grader variability was computed. A software tool was developed to help in this process. The second grader repeated the process $\left(G_{5.2}\right)$ to establish intra-grader variability. The results are shown in Fig. 11, and summarized in Table 2.

The high inter and intra-variability values obtained $(0.020$ and $0.011 \mathrm{~mm}$, respectively), clearly show how difficult the manual process is. 

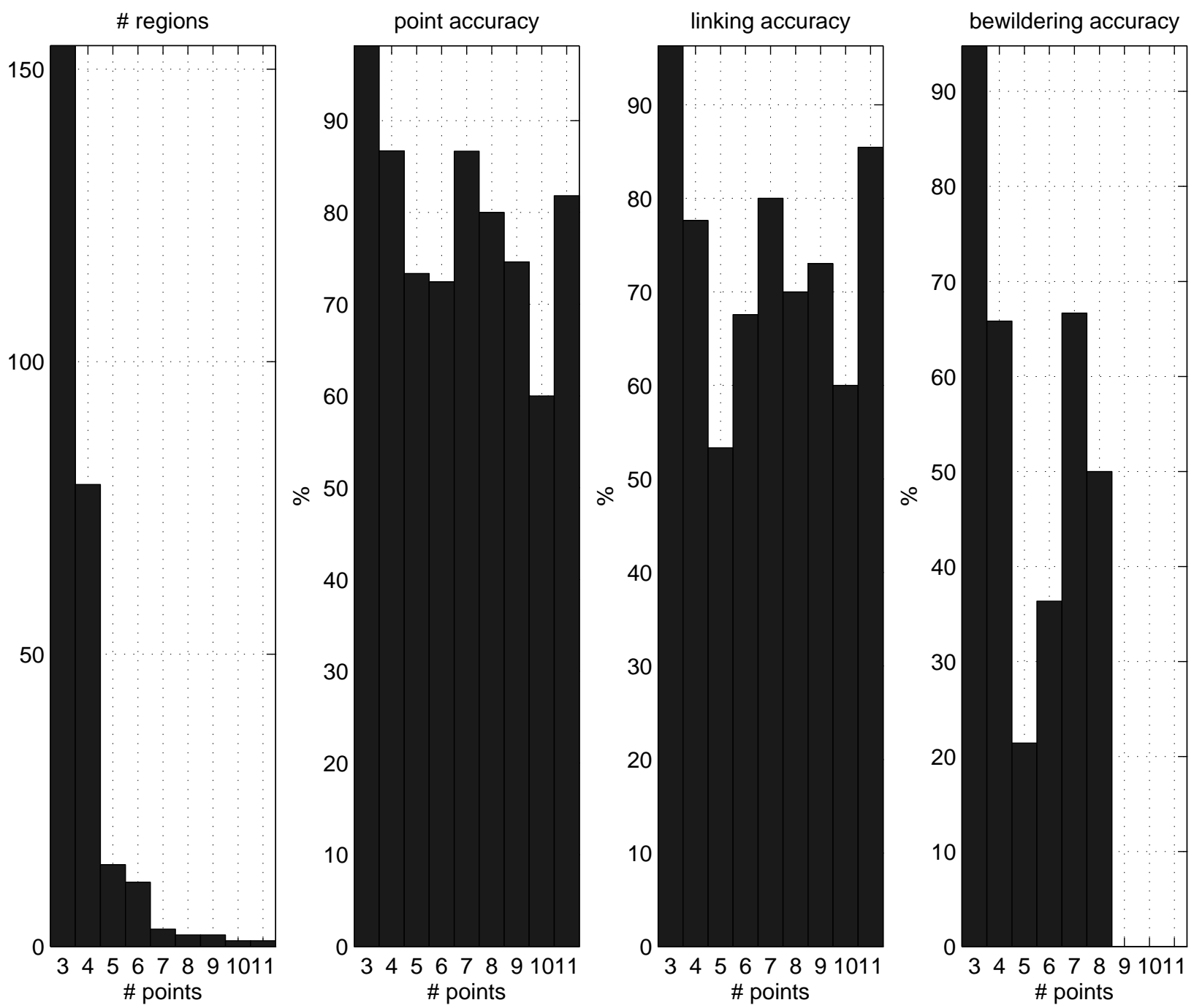

Fig 10 Number of bewildering regions by number of points to link (points in $\mathcal{E}_{c}$ ), on the left, and metric results for bewildering region solving.

Table 2 Comparison between the automatic depth-wise position $(S)$ and each manual grading $\left(G_{4}, G_{5.1}\right.$, and $\left.G_{5.2}\right)$, by the mean and standard deviation (SD) values for the absolute difference $D^{a}(\mathrm{~mm})$ between the result $G$ and $\hat{G}$.

\begin{tabular}{lllll}
\hline Metric & $G$ & $\hat{G}$ & Mean & SD \\
\hline$D^{a}(G, \hat{G})$ & $S$ & $G_{4}$ & 0.013 & 0.009 \\
& $S$ & $G_{5.1}$ & 0.030 & 0.026 \\
& $G_{4}$ & $G_{5.1}$ & 0.020 & 0.021 \\
& $G_{5.2}$ & $G_{5.1}$ & 0.011 & 0.012 \\
\hline
\end{tabular}

As it is visible from Fig. 11, the automatic estimation of the depth-wise position appears mostly at a higher depth than the position estimated by the graders, since the grader aims at the beginning of the shadow (vessel top wall) and the algorithm aims at vessel centerline. Hence, this systematic deviation is expectable. 


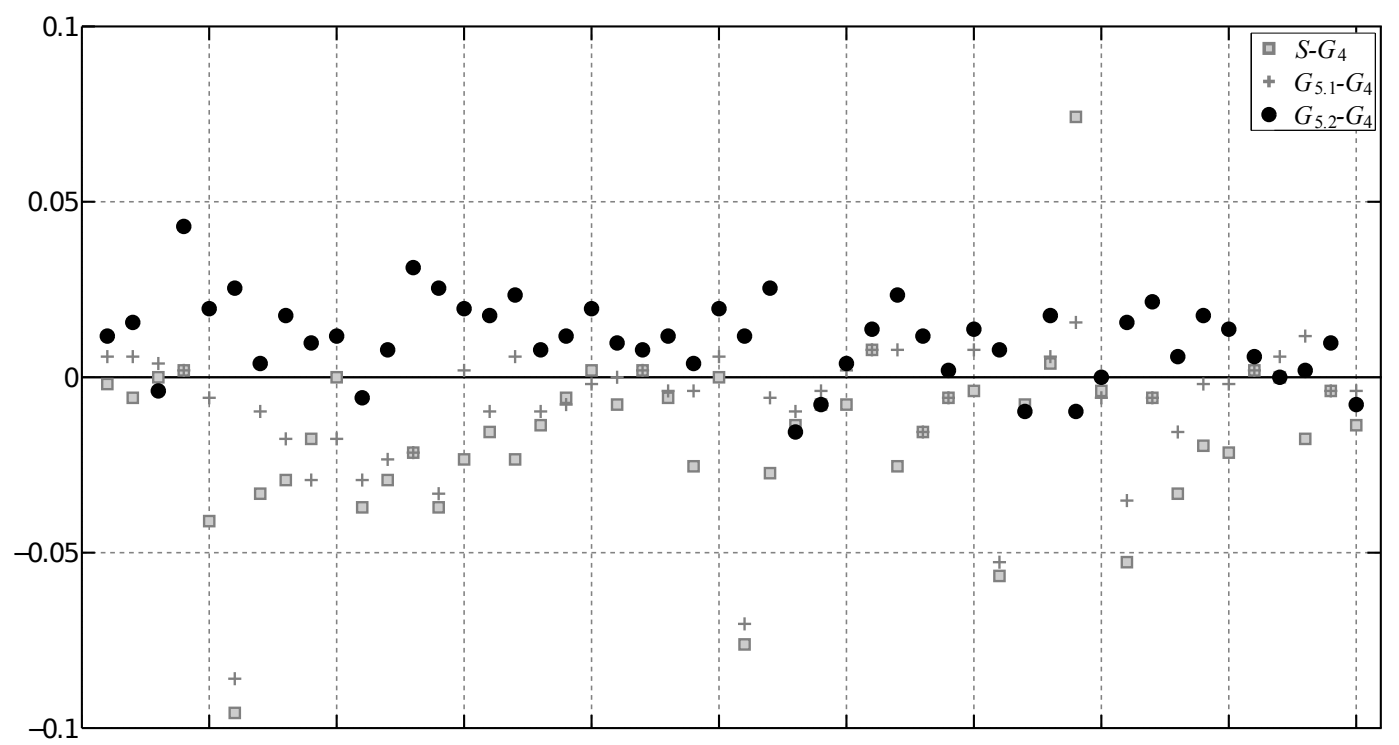

Fig 11 Difference (in milimeters) between the first grader $\left(G_{4}\right)$ manual marking of the beginning of the shadow and the automatic segmentation $(S)$ of the centerline, and both markings of the beginning of the shadow from the second grader $\left(G_{5.1}\right.$ and $\left.G_{5.2}\right)$.
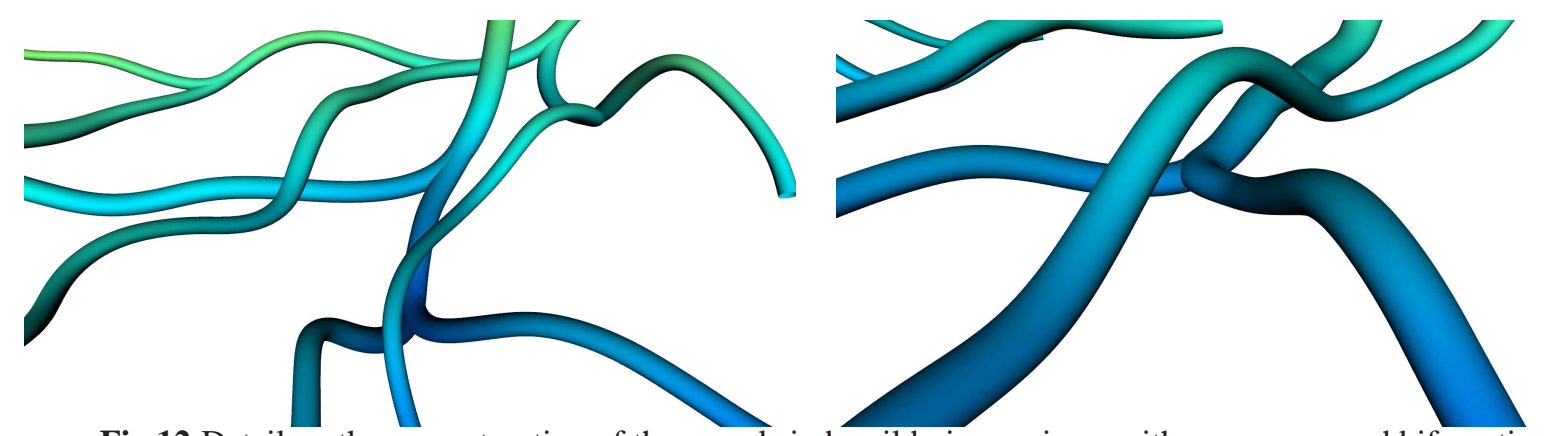

Fig 12 Detail on the reconstruction of the vessels in bewildering regions, with crossovers and bifurcations.

\subsection{Reconstruction}

The proposed reconstruction seems very feasible. Overall, the reconstructed vessel network is smooth and behaves as expected (Fig. 13 and 14). In fact, the details of the crossovers of vessels, as illustrated in Fig. 12, is also according to physicians expectations, where the vessels rapidly deviate to form the crossover. Note that the high axial resolution of OCT is crucial to detect crossovers due to this aspect. Moreover, we illustrate in Fig. 15 the cross-section of the vascular reconstruction in several B-scans. These figures illustrate that the method leads to a feasible reconstruction of the position and shape of the vascular network within the OCT volumetric scan.

From Rodrigues et al. ${ }^{23}$ the execution time for the 2-D segmentation process (OCT fundus reference computation, features computation, and SVM classification), using a MATLAB ${ }^{\circledR}$ (The MathWorks Inc., Natick, MA) implementation, was $65.2 \pm 1.2 \mathrm{~s}(N=15)$. The system hardware used was an Intel ${ }^{\circledR}$ Core $^{\mathrm{TM}}$ i7-3770 CPU (Intel Corporation, Santa Clara, California) at $3.4 \mathrm{GHz}$.

The additional execution time to achieve the 3-D reconstruction using also a MATLAB implementation, was $122.3 \pm 115.1 \mathrm{~s}$ (average \pm standard deviation) on an Intel ${ }^{\circledR}$ Core $^{\mathrm{TM}}$ i7-4770 $\mathrm{CPU}$ at $3.4 \mathrm{GHz}$. For the 3-D reconstruction, the required time greatly depends on the vessel tree 


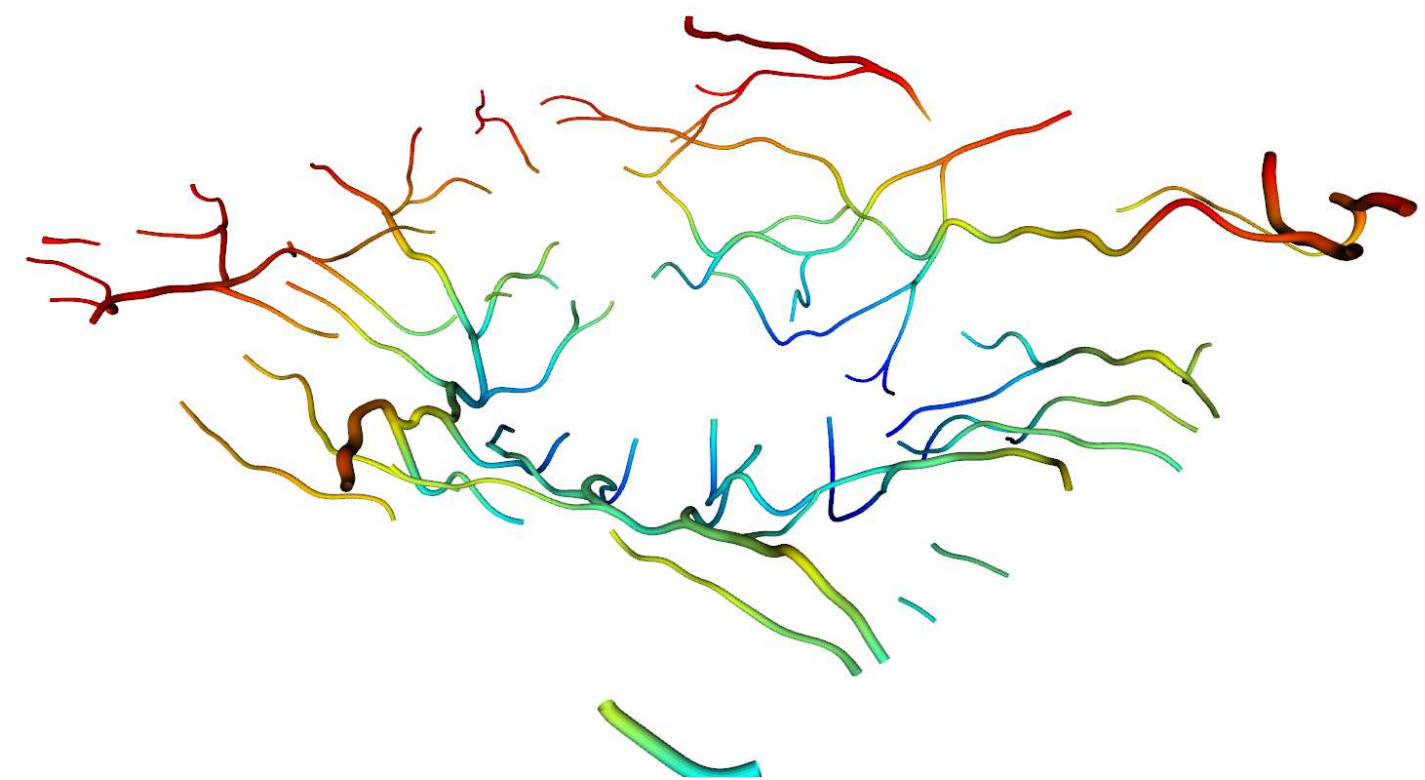

Fig 13 3-D reconstruction of the position and shape of the vessels.

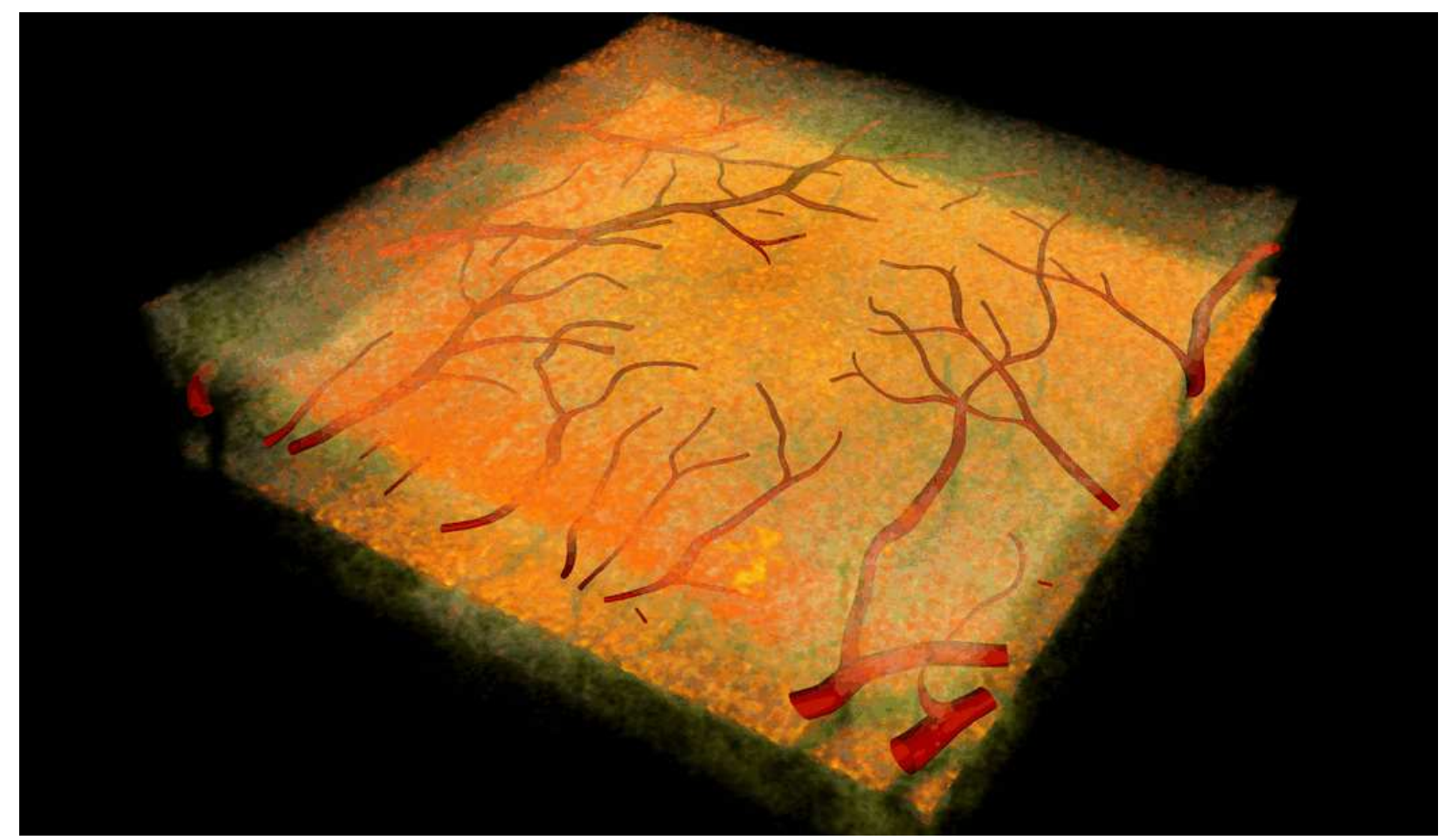

Fig 14 3-D reconstruction of the retinal vascular network embedded in the optical coherence tomography volume.

complexity. The high standard deviation value reflects this behavior. 

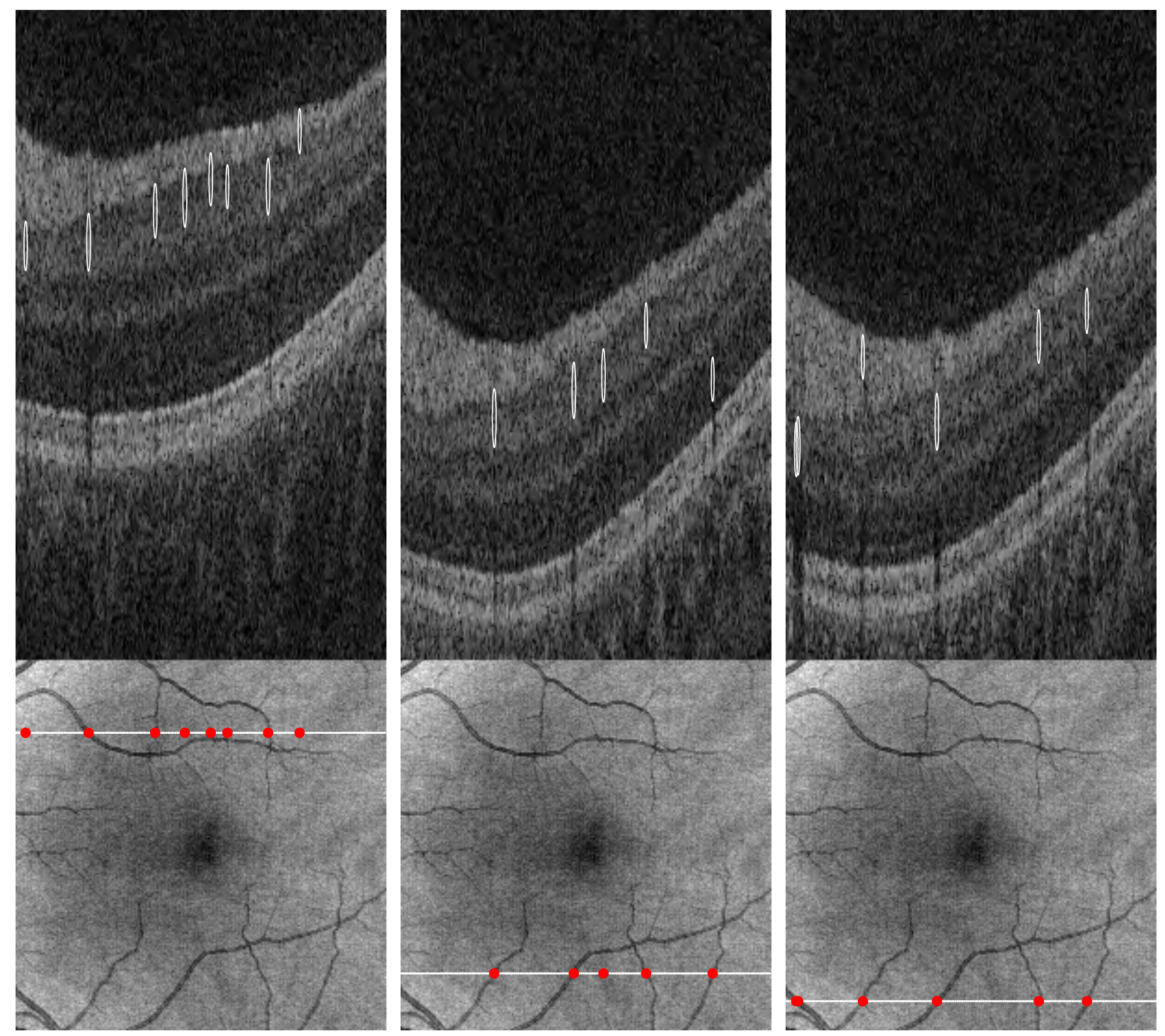

Fig 15 Top: B-scans and cross-section of the vascular reconstruction (in white); Bottom: the respetive position of the B-scan (white line) with the detected vessels (in red) on the fundus reference image.

\section{Discussion and Conclusions}

The method presents a good overall performance. The location of the vascular tree is (as expected) in the upper third of the retina. The vessel calibre estimation achieves a precision similar to a human grader and the depth position detection is in agreement with the known anatomy. However, the linking of the vessels in the defined bewildering regions works well when few connections are involved, but does not seem to contain enough information to achieve higher accuracy when the number of vessel to connect is higher. Apart form the presented cost functions, many other approaches were tried always leading to similar but no better results. Although the bewildering accuracy is about $65 \%$, we note that the linking accuracy and point accuracy are quite high for the problem at hand, having in mind that OCT lateral resolution is low (in comparison with other 
modalities). To the best of our knowledge, this problem is addressed here for the first time for OCT data.

The different steps of the validation show that the algorithm performs well. However, Doppler OCT would be a better ground truth than manual segmentation. Unfortunately, we have no access to such system at our institutions. The high inter and intra-variability from the manual gradings indicate how difficult the manual segmentation is.

The first tests on the relevance of the depth component of the vascular network of the retina have already been conducted. ${ }^{33}$ In this preliminary study, it is shown that the most widely used metric of vessel tortuosity does not have a statistical significant linear relation between 2-D and 3-D metrics. Thus the use of 3-D tortuosity metrics to correlate with disease status might have a significant impact on the correlation values, when compared with those obtained using 2-D metrics.

It is expectable that more severe pathologies would render a more difficult task to overcome. In the future we hope to extend our tests to these cases. Although one might anticipate slightly worst results, please note that our goal of early diagnosis of pathology requires working with eyes within the early stages of disease progression (close to normal).

In the present work, we propose and describe a method to segment and visualize the retinal vascular network in 3-D for OCT data. Increased sampling and accuracy would improve the algorithms performance and would allow for an objective validation.

\section{Acknowledgment}

This work was supported by FCT under the research projects PTDC/SAU-ENB/111139/2009 and PEST-C/SAU/UI3282/2013, and by the COMPETE programs FCOMP-01-0124-FEDER-015712 and FCOMP-01-0124-FEDER-037299. The authors would like to thank António Correia for performing the manual gradings.

\section{References}

1 P. Guimarães, P. Rodrigues, P. Serranho, and R. C. Bernardes, "3D Retinal Vascular Network from Optical Coherence Tomography Data," in Image Analysis and Recognition, A. Campilho and M. Kamel, Eds., Lecture Notes in Computer Science, 339-346, Springer Berlin Heidelberg (2012).

2 G. Liew, J. J. Wang, P. Mitchell, and T. Y. Wong, "Retinal vascular imaging: a new tool in microvascular disease research," Circulation. Cardiovascular imaging 1, 156-61 (2008).

3 R. Kawasaki, N. Cheung, T. Mosley, F. M. A. Islam, A. R. Sharrett, R. Klein, L. H. Coker, D. S. Knopman, D. K. Shibata, D. Catellier, and T. Y. Wong, "Retinal microvascular signs and 10-year risk of cerebral atrophy: the Atherosclerosis Risk in Communities (ARIC) study," Stroke 41, 1826-8 (2010).

4 N. Witt, T. Y. Wong, A. D. Hughes, N. Chaturvedi, B. E. K. Klein, R. Evans, M. McNamara, S. A. M. Thom, and R. Klein, "Abnormalities of retinal microvascular structure and risk of mortality from ischemic heart disease and stroke," Hypertension 47, 975-81 (2006).

5 F. N. Doubal, T. J. MacGillivray, N. Patton, B. Dhillon, M. S. Dennis, and J. M. Wardlaw, "Fractal analysis of retinal vessels suggests that a distinct vasculopathy causes lacunar stroke," Neurology 74, 1102-7 (2010). 
6 R. Kawasaki, J. Xie, N. Cheung, E. Lamoureux, R. Klein, B. E. K. Klein, M. F. Cotch, A. R. Sharrett, S. Shea, and T. Y. Wong, "Retinal microvascular signs and risk of stroke: the Multi-Ethnic Study of Atherosclerosis (MESA)," Stroke 43, 3245-51 (2012).

7 N. Patton, T. M. Aslam, T. J. MacGillivray, A. Pattie, I. J. Deary, and B. Dhillon, "Retinal vascular image analysis as a potential screening tool for cerebrovascular disease: a rationale based on homology between cerebral and retinal microvasculatures," Journal of anatomy 206, 319-48 (2005).

8 N. Patton, A. Pattie, T. J. MacGillivray, T. M. Aslam, B. Dhillon, A. Gow, J. M. Starr, L. J. Whalley, and I. J. Deary, "The association between retinal vascular network geometry and cognitive ability in an elderly population," Investigative ophthalmology \& visual science $\mathbf{4 8}$, 1995-2000 (2007).

9 H. Yatsuya, A. R. Folsom, T. Y. Wong, R. Klein, B. E. K. Klein, and A. R. Sharrett, "Retinal microvascular abnormalities and risk of lacunar stroke: Atherosclerosis Risk in Communities Study," Stroke 41, 1349-55 (2010).

10 P. Z. Benitez-Aguirre, M. B. Sasongko, M. E. Craig, A. J. Jenkins, J. Cusumano, N. Cheung, T. Y. Wong, and K. C. Donaghue, "Retinal vascular geometry predicts incident renal dysfunction in young people with type 1 diabetes," Diabetes care 35, 599-604 (2012).

11 X. Wang, H. Cao, and J. Zhang, "Analysis of retinal images associated with hypertension and diabetes," in Proceedings of the 27th Annual International Conference of the IEEE Engineering in Medicine and Biology Society, 6, 6407-10, IEEE (2005).

12 M. B. Sasongko, J. J. Wang, K. C. Donaghue, N. Cheung, P. Z. Benitez-Aguirre, A. J. Jenkins, W. Hsu, M.-L. Lee, and T. Y. Wong, "Alterations in retinal microvascular geometry in young type 1 diabetes," Diabetes care 33, 1331-6 (2010).

13 B. E. Bouma, G. J. Tearney, et al., Handbook of optical coherence tomography, Marcel Dekker New York: (2002).

14 R. Bernardes and J. Cunha-Vaz, Optical Coherence Tomography: A Clinical and Technical Update, Springer (2012).

15 T. Fabritius, S. Makita, Y. Hong, R. Myllylä, and Y. Yasuno, "Automated retinal shadow compensation of optical coherence tomography images," Journal of biomedical optics 14(1), 010503 (2009).

16 W. Drexler and J. G. Fujimoto, Optical coherence tomography: technology and applications, Springer (2008).

17 M. Niemeijer, M. K. Garvin, B. van Ginneken, M. Sonka, and M. D. Abràmoff, "Vessel Segmentation in 3D Spectral OCT Scans of the Retina," in Proc. SPIE 6914, Medical Imaging 2008: Image Processing, J. M. Reinhardt and J. P. W. Pluim, Eds., 6914, 69141R (2008).

18 J. Xu, D. A. Tolliver, H. Ishikawa, G. Wollstein, and J. S. Schuman, "3D OCT retinal vessel segmentation based on boosting learning," in World Congress on Medical Physics and Biomedical Engineering, September 7 - 12, 2009, Munich, Germany, O. Dössel and W. C. Schlegel, Eds., IFMBE Proceedings, 179-182, Springer Berlin Heidelberg (2009).

19 R. Kafieh, H. Danesh, H. Rabbani, M. D. Abràmoff, and M. Sonka, "Vessel segmentation in images of optical coherence tomography using shadow information and thickening of Retinal Nerve Fiber Layer," in 2013 IEEE International Conference on Acoustics, Speech and Signal Processing, 1075-1079, IEEE (2013). 
20 M. Pilch, Y. Wenner, E. Strohmayr, M. Preising, C. Friedburg, E. M. zu Bexten, B. Lorenz, and K. Stieger, "Automated segmentation of retinal blood vessels in spectral domain optical coherence tomography scans," Biomedical optics express 3, 1478-91 (2012).

21 Z. Hu, M. Niemeijer, M. D. Abràmoff, K. Lee, and M. K. Garvin, "Automated segmentation of 3-D spectral OCT retinal blood vessels by neural canal opening false positive suppression," in Medical Image Computing and Computer-Assisted Intervention-MICCAI 2010, T. Jiang, N. Navab, J. P. W. Pluim, and M. A. Viergever, Eds., Lecture Notes in Computer Science 13, 33-40, Springer Berlin Heidelberg (2010).

22 V. Kajić, M. Esmaeelpour, C. Glittenberg, M. F. Kraus, J. Honegger, R. Othara, S. Binder, J. G. Fujimoto, and W. Drexler, "Automated three-dimensional choroidal vessel segmentation of 3d $1060 \mathrm{~nm}$ oct retinal data," Biomedical optics express 4(1), 134-150 (2013).

23 P. Rodrigues, P. Guimarães, T. Santos, S. Simão, T. Miranda, P. Serranho, and R. C. Bernardes, "Two-dimensional segmentation of the retinal vascular network from optical coherence tomography," Journal of biomedical optics 18, 126011 (2013).

$24 \mathrm{~K} . \mathrm{Li}, \mathrm{X} . \mathrm{Wu}, \mathrm{D}$. Z. Chen, and M. Sonka, "Optimal surface segmentation in volumetric images - a graph-theoretic approach," IEEE transactions on pattern analysis and machine intelligence 28, 119-34 (2006).

25 P. A. Dufour, L. Ceklic, H. Abdillahi, S. Schröder, S. De Dzanet, U. Wolf-Schnurrbusch, and J. Kowal, "Graph-based multi-surface segmentation of OCT data using trained hard and soft constraints," IEEE transactions on medical imaging 32, 531-43 (2013).

26 P. Guimarães, P. Rodrigues, C. a. L. Lobo, S. Leal, J. a. Figueira, P. Serranho, and R. C. Bernardes, "Ocular Fundus Reference Images from Optical Coherence Tomography," Computerized Medical Imaging and Graphics (2014).

27 B. Al-Diri, A. Hunter, and D. Steel, "An active contour model for segmenting and measuring retinal vessels," IEEE transactions on medical imaging 28, 1488-97 (2009).

28 X. Xu, M. Niemeijer, Q. Song, M. Sonka, M. K. Garvin, J. M. Reinhardt, and M. D. Abràmoff, "Vessel boundary delineation on fundus images using graph-based approach," IEEE transactions on medical imaging 30, 1184-91 (2011).

29 E. Trucco, L. Ballerini, D. Relan, A. Giachetti, T. J. MacGillivray, K. Zutis, C. Lupascu, D. Tegolo, E. Pellegrini, G. Robertson, P. J. Wilson, A. Doney, and B. Dhillon, "Novel VAMPIRE algorithms for quantitative analysis of the retinal vasculature," in 2013 ISSNIP Biosignals and Biorobotics Conference (BRC), 1-4, IEEE (2013).

30 B. Al-Diri, A. Hunter, D. Steel, and M. Habib, "Manual measurement of retinal bifurcation features.," in 2010 Annual International Conference of the IEEE Engineering in Medicine and Biology Society, 2010, 4760-4 (2010).

31 B. Al-Diri, A. Hunter, D. Steel, and M. Habib, "Automated analysis of retinal vascular network connectivity," Computerized Medical Imaging and Graphics 34(6), 462-470 (2010).

32 J. a. V. B. Soares, J. J. G. Leandro, R. M. Cesar, H. F. Jelinek, and M. J. Cree, "Retinal vessel segmentation using the 2-D Gabor wavelet and supervised classification," IEEE Transactions on Medical Imaging 25, 1214-1222 (2006).

33 P. Serranho, P. Guimarães, P. Rodrigues, and R. C. Bernardes, "On the relevance of the 3D retinal vascular network from OCT data," Biometrical Letters 49, 95-102 (2012). 


\section{List of Figures}

1 Flowchart representing the global workflow of the algorithm. . . . . . . . . . .

2 Projection of a 3-D macular OCT scan to a 2-D fundus reference image by mapping the shadows casted due to light absorption. The binary image (from the classification of the pixels in the 2-D reference) is then used to aid in the identification of the depth location of the vessels. . . . . . . . . . . . . . . . . . .

3 Flowchart for the 3-D segmentation block of Fig. 1. The main block inputs are the OCT volume, the segmented layers, the 2-D ocular fundus reference image, and the binary image $($ Fig. 2$) \ldots \ldots \ldots \ldots$. . . . . . . . . . . . . 4

4 Examples of 2-D vessel binary maps cropped to bewildering regions of crossovers and/or bifurcations. . . . . . . . . . . . . . . . . .

5 Detail of a principal component fundus reference image (a) and the respective binary maps of the 2-D classification $\mathcal{U}$ (b) and of the vessel centrelines (with the removed bewildering regions in red) $\mathcal{U}^{\text {skel }}(\mathrm{c}) . \ldots \ldots \ldots$

6 Computation examples of the depth coordinate of different blood vessel at a centreline point $i$. The two profiles on the top of each plot are the vessel profile $A_{i}^{\text {vessel }}$ (blue) and the non-vessel profile $A_{i}^{\text {non-vessel }}$ (red). The difference between the two (black profile) is shown on the bottom. The difference profile is filtered at the region of interest (green) and the depth coordinate of the vessel on that point is taken as the location of the maximum of the filtered difference. . . . . . . . . . . .

7 Illustration of a local reconstruction of a vessel, based on Delaunay triangulation. Several points over the centreline are chosen and equally distant points (with distance equal to the estimated radius of the vessel in the $x y$ plane) inn the respective orthogonal plane to the centreline are considered for the 3-D triangulationprocess of reconstruction. . . . . . . . . . . . . . . . . . .

8 Average relative differences for the automatic estimation $(S)$ and manual gradings $\left(G_{1}, G_{2}\right.$, and $\left.G_{3}\right)$ displayed by calibre range. . . . . . . . . . . . . . . 11

9 Representative results for the diameter estimation. The manual gradings are marked with (white) $\vdash \dashv$ and automatic estimation is marked with the (yellow) dotted line.

10 Number of bewildering regions by number of points to link (points in $\mathcal{E}_{c}$ ), on the left, and metric results for bewildering region solving. . . . . . . . . . . .

11 Difference (in milimeters) between the first grader $\left(G_{4}\right)$ manual marking of the beginning of the shadow and the automatic segmentation $(S)$ of the centerline, and both markings of the beginning of the shadow from the second grader $\left(G_{5.1}\right.$ and

12 Detail on the reconstruction of the vessels in bewildering regions, with crossovers and bifurcations. . . . . . . . . . . . . . . . . . . 14

13 3-D reconstruction of the position and shape of the vessels. . . . . . . . . 15

$143-D$ reconstruction of the retinal vascular network embedded in the optical coherence tomography volume. . . . . . . . . . . . . . . . . 15

15 Top: B-scans and cross-section of the vascular reconstruction (in white); Bottom: the respetive position of the B-scan (white line) with the detected vessels (in red) on the fundus reference image. . . . . . . . . . . . . . . . . . 16 